\title{
LA HUELGA GENERAL PROLETARIA: UN CAMINO HACIA «EL VERDADERO ESTADO DE EXCEPCIÓN» (DISCUSIONES TEÓRICAS)
}

Cristian Ignacio Vidal Barría

\begin{abstract}
RESUMEN
Walter Benjamin, en la tesis VIII de su texto sobre el concepto de historia (T. Adorno intituló de esa manera el texto), señala que la tradición de los oprimidos nos permite advertir que vivimos en un "estado de excepción» que es la regla. Al respecto Benjamin propone «promover el verdadero estado de excepción» que desarticule la «norma histórica» que avanza en nombre del progreso. De esa manera el oprimido dejará de ser parte de una estructura binaria en la que siempre su lugar es la del vencido. A partir de estas reflexiones hemos de proponer un camino hacia «el verdadero estado de excepción» y para ello indagamos en la posibilidad de que este sea a través de la denominada «Huelga General Proletaria» en la que se detiene Benjamin en uno de sus textos temprano: Para una crítica de la violencia (1920).
\end{abstract}

Palabras Claves: Huelga General, Estado de excepción, Tesis VIII, Historia.

\section{THE PROLETARIAN GENERAL STRIKE: A PATH TOWARDS “THE TRUE STATE OF EXCEPTION" (THEORETICAL DISCUSSIONS)}

\begin{abstract}
Walter Benjamin, in the thesis VIII of his text On the concept of history (T. Adorno entitled in this way the text), points out that the tradition of the oppressed allows us to notice that we live in a "state of exception" which is a rule. In this regard, Benjamin proposes «promoting the true state of exception», which dismantles the "historical norm» that advances in the name of progress. In this way, the oppressed will no longer be part of a binary structure in which his place is always that of the vanquished. From these reflections, we must propose a path towards "the true state of exception" and for this we research the possibility that this might be through the so-called "Proletarian General Strike", in which Benjamin stops in one of his early texts: Critique of Violence (1920).
\end{abstract}

KEYWORDS: General Strike, State of Exception, Thesis VIII, History. 
VIII

"La tradición de los oprimidos nos enseña que el "estado de excepción" en que ahora vivimos es en verdad la regla. El concepto de historia al que lleguemos debe resultar coherente con ello. Promover el verdadero estado de excepción se nos presentará entonces como tarea nuestra, lo que mejorará nuestra posición en la lucha contra el fascismo. La oportunidad que éste tiene está, en parte no insignificante, en que sus adversarios lo enfrentan en nombre del progreso como norma histórica. El asombro ante el hecho de que las cosas que vivimos sean "aún" posibles en el siglo veinte no tiene nada de filosófico. No está al comienzo de ningún conocimiento, a no ser el de que la idea de la historia de la cual proviene ya no puede sostenerse."

(Walter Benjamin, trad. De Bolívar Echeverría)

\section{Sobre el estado de excepción y el verdadero estado de excepción: diálogos entre Benjamin y Schmitt}

El texto póstumo de Walter Benjamin, Las tesis sobre el concepto de historia, se construye a partir de una serie de reflexiones que pueden ser leídas y agrupadas como críticas a diversos temas que se ven, incluso, en trabajos anteriores de este filósofo alemán. Lo evidente en los fragmentos sobre la historia es la dimensión política que propone Benjamin para la reflexión sobre temas como la historia oficial, el progreso, el positivismo u otros que se conectan entre sí. Una idea en particular, presente en las tesis sobre el concepto de historia, es la que pretendemos revisar en estas hojas. Se trata de la idea de estado de excepción que introduce Benjamin en uno de los fragmentos y que, a nuestro entender, establece un diálogo con el texto temprano del autor: Para una crítica de la violencia.

Señala Benjamin, en la tesis VIII a propósito de los oprimidos, que vivimos en un «estado de excepción» que es la regla y que nuestra «tarea» es producir un «verdadero estado de excepción». Siguiendo lo que Benjamin advierte, hemos de preguntarnos por cuál es el verdadero estado de excepción al que deberíamos 
llegar. $\mathrm{O}$, más bien, de qué manera podemos, siquiera, encontrar un espacio que nos permita acercarnos a ese estado; solo de esta manera se podrá concebir «un nuevo concepto de historia», que se corresponda con la tradición de los oprimidos -nos dirá Benjamin. Frente a esta problemática planteamos como hipótesis que un camino para llegar al verdadero estado de excepción es la denominada Huelga General Proletaria, que es revisada por Benjamin en el libro de 1920 Para una crítica de la violencia. Nuestro interés, por lo tanto, es indagar, en principio, en el concepto de «estado de excepción» que integra Benjamin en la tesis VIII, para luego revisar y establecer un diálogo entre este y la noción de huelga, trabajada por George Sorel en Reflexiones sobre la violencia.

En principio nos gustaría señalar la dimensión aparentemente dicotómica que queda al descubierto tanto en la tesis VIII como en otras del mismo ensayo. Lo que sugiere su autor es, como bien lo anticipamos, poner de manifiesto la articulación de un tipo de historia que solo se ocupa de los vencedores y que, por lo tanto, deja a «los oprimidos» en un permanente estado de excepción. Esta historia de los vencedores logra sobreponerse ya que avanza con la bandera del progreso. Frente a esta realidad la propuesta de Benjamin es encaminarse a un nuevo concepto de historia en donde tengan espacio los oprimidos, de manera que «pueda mejorar la lucha contra el fascismo " ${ }^{1}$. Esta alternativa solo es, en apariencia dicotómica, pues no se trata de que se intercambien los roles y ahora se escriba la historia de los oprimidos por sobre la de los vencedores. Es decir, no basta con una «reforma» de la historia, sino que, al contrario, se trata de encontrar otros espacios que se abran por donde encaminar el verdadero estado de excepción.

Sin embargo, antes de llegar a ese estadio es necesario trabajar sobre la idea de estado de excepción, lo que nos lleva hasta la concepción que tiene «el jurista del Tercer Reich", Carl Schmitt, sobre esto. No cabe duda que Benjamin es un conocedor del pensamiento de Schmitt, en particular del libro Teología política

\footnotetext{
${ }^{1}$ En uno de los textos preparatorios de las tesis Benjamin señala que se necesita una teoría de la historia sobre cuya base el fascismo sea desenmascarado.
} 
$(1922)^{2}$. En este se plantea la relación entre soberanía y estado de excepción. Para Schmitt «Soberano es el que decreta el estado de excepción» (Schmitt, 1934, 11), un estado que suspende todo el orden existente, creando derecho y convirtiendo su decisión en ley. Sin embargo, esta suspensión del orden no debe pensarse desde una perspectiva en que «el suspendido quede libre de toda norma o desligado del derecho» (Mate 2006, 146), pues esa idea, más bien, es la que sugiere Benjamin cuando se refiere al verdadero estado de excepción. Lo que señala aquí Schmitt es que el individuo queda bajo el poder directo del soberano sin que en esta relación exista mediación alguna, llámese derecho, norma o ley. Reyes Mate lo señala de la siguiente manera:

Para un jurista como Carl Schmitt, obsesionado por el vértigo del caos social, el estado de excepción le venía como anillo al dedo porque refuerza la autoridad del poder al ubicar su legitimación en la pura decisión del soberano. El poder de suspender las leyes nos descubre el secreto del poder político, que no es aplicar leyes ni hacer leyes por caminos reglados, sino convertir su voluntad en ley. (MATE, 2006, 147)

Giorgio Agamben, en Homo Sacer, ha referido la complejidad que supone la idea del estado de excepción. Por un lado, señala, que el estado de excepción se funda en una necesidad que "no puede revestir forma jurídica» (AGAMBEN, 2010, 9), pues este se presentaría como la paradoja de una «forma legal de algo que no puede tener forma legal». Es evidente que lo que señala Agamben toma un camino distinto a lo que en las tesis de la historia señala Benjamin. Agamben propone una lectura contemporánea, advirtiendo los problemas que suscita la idea de estado de excepción; todo esto inserto dentro de un sistema político y jurídico establecido. Por su parte, Benjamin busca desarticular este sistema establecido y para ello recurre al estado de excepción (devenido en regla) como una de las razones que justifican esta postura. No obstante, en alguna medida ambas miradas se cruzan y dialogan, pues Agamben también advierte que «este no solo se presenta cada vez más como una técnica de gobierno» (AGAMBEN, 2010, 17), al contrario, deja al descubierto

\footnotetext{
${ }^{2}$ Benjamin cita a Schmitt en su libro sobre el drama barroco alemán. También le escribe una carta en indicándole que le enviará este mismo libro.
} 
su forma paradigmática como «constitutiva del orden jurídico». Es decir, deja al descubierto su conversión en una «regla».

Ahora bien, cuando Benjamin habla del verdadero estado de excepción señala que necesitamos de este para tener una mejor posición en la lucha contra el fascismo. Sin embargo, en consonancia con los demás fragmentos de la historia, podemos esgrimir que no se trata tan solo de una «lucha contra el hitlerismo» pues — como señala Mate- «si el sujeto de la excepcionalidad fuera el régimen nazi, las cosas serían más fáciles, pues bastaría con vencerle para acabar con ese estado de cosas» (MATE, 2006, 149). Entonces, cuando Benjamin se refiere al fascismo, no lo hace de modo directo a un tipo de régimen ${ }^{3}$, sino que a todos quienes propician que este pueda acontecer, entre ellos, claramente a quienes escriben y reproducen una forma de historia. Tampoco debemos asombrarnos que en el siglo Xx existan regímenes nazis. Es decir, no tiene objeto tomar esta realidad como hechos anacrónicos (pensar el fascismo como lo antiguo), si para que esta realidad surgiera se necesitó de elementos que solo la modernidad pudo ofrecer. Lo que en realidad se debe advertir es el hecho de que, al convertirse en regla, el estado de excepción pasaría, eventualmente y de modo gradual, a establecerse y reemplazar al estado de derecho (se difumina la noción de estado de excepción y de derecho), pero que funciona a través de los derechos que el soberano instaura para ejercer el poder y la violencia sobre quienes han sido históricamente avasallados.

Las diferencias entre Schmitt y Benjamin respecto al estado de excepción, son evidentes; en el mismo espacio en que en el «sentido schmittiano, hay fuerza

\footnotetext{
${ }^{3}$ No quiere decir que la violencia desplegada en los regímenes fascistas no se constituya como parte de la regla. Según nuestra lectura, son estos momentos en que, con mayor claridad, percibimos como opera el estado de excepción devenido en regla para los oprimidos. Hay quienes han estudiado el estado de excepción en directa relación con el concepto de "campo». Por ejemplo, Kamal Cumsille lo señala de la siguiente manera: «la segunda guerra, con el fenómeno del fascismo, donde el totalitarismo como principio según el cual "todo es posible", convierte al "estado de excepción" en la norma del devenir político, e instala al campo de concentración como el espacio en donde este devenir tiene lugar». Es decir, el campo de concentración se constituye como el lugar originario donde emerge el estado de excepción. Agamben, por su parte dirá "El campo es el espacio que se abre cuando el estado de excepción comienza a devenir la regla".

En otro estudio se señala que la manifestación actual del estado de excepción convertido en regla y en relación con el campo, se da en la cárcel de Guantánamo. Este sería un lugar en donde las personas carecen de derecho o leyes y no siempre por causas bien esclarecidas.
} 
de ley sin ley», Benjamin solo ve «violencia conservadora» convertida en regla. «Allí donde Schmitt observa la mano visible que alumbra la salida, Benjamin ve el azote que cercena la escapatoria» (LUCCA, 2009, 103). Esta violencia - señala Benjamin - se encuentra monopolizada y entabla algún diálogo con el estado de excepción que, como señalamos antes, se difumina con el estado de derecho. El temor, en cuanto a la violencia, se da cuando este «no está en las manos del derecho correspondiente, lo pone en peligro, no por los fines que pueda perseguir, sino por su mera existencia fuera del derecho» $(2006,5)^{4}$. Por lo tanto, desde la perspectiva de Benjamin, es esta excepción la que se debe eliminar ya que "conlleva la suspensión del derecho», pero a la vez la integración de unas nuevas formas de derecho que se convertirán en regla.

Valga la repetición para reafirma que cuando Benjamin se refiere a un estado de excepción verdadero, este no supone establecer un decreto nuevo, por sobre otro. Es más bien un cuestionamiento sobre el botín de guerra que llevan los vencedores (y que de esa manera se ha constituido durante siglo): ¿acaso ese botín no tiene más utilidad que dar validez a quiénes vencieron, todo esto en pos del progreso?: a la luz de una larga hilera de muertos que ha quedado en el camino. $O$ bien, son aquellos oprimidos, parte de una estructura binaria de vencedor/vencido cuyo lugar en la historia es únicamente para que exista un vencedor. Es evidente que lo planteado por Benjamin responde a la negación de esta forma de hacer la historia y sugiriendo la búsqueda de un espacio de resistencia que origine o germine la producción de una nueva forma de la historia.

\section{El trayecto de la huelga a la huelga general: articulaciones de la violencia}

Planteamos aquí que esa forma o espacio desde donde se propicia un camino que nos lleve a un estado de excepción verdadero es por medio de la llamada Huelga General Proletaria. Tanto Sorel como Benjamin diferencian a esta

\footnotetext{
${ }^{4}$ Citamos la traducción de Pablo Oyarzun del año 2006 del libro de Benjamin Para una crítica de la violencia originalmente publicado en el año 1921.
} 
de otros tipos de huelga pues no todas poseen las mismas características. Antes de señalar la distinción de estos dos tipos de huelga es necesario retomar, y añadir, la noción de violencia ${ }^{5}$ que circunda todo el entramado que queremos presentar. Señalamos en el párrafo anterior que, en el estado de excepción, Benjamin ve un tipo de «violencia conservadora», monopolizado en principio por el estado y luego por el estado de excepción que ha reemplazado al estado de derecho (al menos esa es la lectura que nosotros hacemos). Sin embargo, emerge la figura del derecho a huelga que, para Benjamin, es la única figura a la que se le otorga un derecho a la violencia por parte del estado. Asumiendo, claro, la huelga como una instancia violenta en la medida que irrumpe una «armonía» o forma establecida en que se desenvuelve un sistema. Sin embargo, para el estado, con el derecho a huelga no está permitido, en ningún caso, un derecho a la violencia «sino más bien un derecho a sustraerse de ella». Pues, según el estado la «abstención de actuar», significa un «no hacer» que, en definitiva, refleja «aquello en que consiste la huelga [y] no puede en modo alguno caracterizarse como violencia» (BENJAMIN, 2006, 5). Esto da pie, según Benjamin, a que frente a cualquier indicio de violencia se suprima el derecho a la huelga lo que, de modo paradójico, significa ahora un tipo de violencia por parte del estado; esto en cuanto se desarticula una manifestación legítima. Benjamin lo dirá en los siguientes términos:

[...] el momento violento aparece necesariamente en dicha abstención, y en forma de chantaje, cuando se presenta en la disposición de principio a volver a ejercitar la actividad interrumpida bajo ciertas condiciones que nada tienen que ver con ella o bien que solo modifican algo exterior en ella. $Y$ en este sentido el derecho a huelga constituye, desde la perspectiva de los trabajadores, que se enfrenta a la del Estado, el derecho de utilizar la violencia para llevar a efecto determinados fines $(2006,6)$

\footnotetext{
${ }^{5}$ Acercarnos al concepto «violencia» nos resulta problemática en la medida que en alemán esta palabra nos remite tanto a la violencia como al poder. Conceptos que, por lo demás, se encuentran estrechamente relacionados. De esa manera cuando referimos este concepto con la idea de poder bien podemos estar hablando del poder (o la violencia) de Dios que en este caso no es ejercida bajo la concepción actual que tenemos de violencia sino más bien como un tipo de poder autoritario. Por un tema funcional, y por la naturaleza de este ensayo, hemos de tomar el concepto alemán Gewalt con el «significado fundamental» (dirá Oyarzun), con el que la emplea Benjamin.
} 
Como bien señalamos, resulta paradójico el derecho a huelga que otorga el estado ya que este le subyace un interés que, de ningún modo se relaciona con otorgar mayor derecho a los trabajadores. Se trata, más bien, de «generar un marco legal para distinguir las huelgas que reivindican demandas económicas negociables y sancionar el desborde de una huelga general con reivindicaciones revolucionarias» (PÉREZ LÓPEZ, 2016, 84). Tales sanciones no necesariamente se manifiestan en violencia física, la que, dicho sea de paso, solo corroboraría el interés subyacente que aquí esbozamos. Estas, cabe reafirmar, se dan como actos violentos al tener que «encausar» la huelga nuevamente hacia el trabajo habiendo llegado, en el mejor de los casos, a cierto acuerdo laboral. Es decir, la posición del estado es, por una parte, llegar a un acuerdo o reprimir. Es evidente que ninguna tiene lógica y carecen de ética, pues ha sido el mismo estado quien ha puesto las reglas de una situación de la que es parte y al no verse favorecido actúa en un nuevo marco excepcional.

Lo que acabamos de señalar define de alguna manera un tipo de huelga que no llega a configurarse como la Huelga General Proletaria, debido a que sufre su desarticulación por medio de la violencia estatal y con ella puede no conseguir nada o bien conseguir «mejoras» salariales o laborales. Estas mejoras no implican ni nos llevan por el camino que determine el verdadero estado de excepción, pero pueden ser leídas como una sucesión de momentos que pugnan contra la historia del progreso y logran «torcer» el camino de esta, mas no llevar la historia a contrapelo o a su desarticulación. Incluso, en esta dinámica queda al descubierto la «norma histórica» a la que Benjamin se referirá en la tesis que en esta revisión nos ocupa.

La huelga general, por otro lado, no se puede concebir como un derecho adquirido en que el estado la concede y la retrotrae (la regula) en cuanto advierte la magnitud y las consecuencias que puede traer esta. Para Benjamin, en la huelga general «los trabajadores reclamarán siempre su derecho a huelga, mientras que el Estado señalará ese reclamo como un abuso, porque el derecho a huelga no se ha concebido "asi", y promulgará sus medidas extraordinarias", o excepcionales. Frente a estas medidas excepcionales la huelga general continuará su rearticulación, pues uno de sus intereses será configurar una experiencia 
revolucionaria que pueda alterar una «norma histórica» y de esa manera desbordar aquellos «mecanismo jurídicos dispuestos por el Estado, para negociar meras demandas laborales» (PÉREZ LÓPEZ, 2016, 80). Para Georges Sorel los dos tipos de huelgas son, en concreto, una de carácter político y una proletaria ${ }^{6}$. En la política, se fortalece la violencia estatal. Dirá Sorel que esta «demuestra cómo el Estado no perderá nada de su fuerza, cómo se traspasa el poder de privilegiados a privilegiados, cómo la masa de los productores trocará sus amos.» (SOREL, 1979, 265). En el otro lado, para Sorel, emerge la huelga general proletaria, la que, según nuestra hipótesis se correspondería con la búsqueda del verdadero estado de excepción que debemos producir (suscitar, procurar).

Ahora bien, más allá de lo que hemos sugerido, la pregunta que debemos responder es sobre las características de la huelga general proletaria que la posicionan — parafraseando a Derrida - como una tentación deconstructiva ${ }^{7}$ del estado de excepción devenido en regla. Sorel en Reflexiones sobre la violencia, señala que una de las tareas de la huelga general proletaria es la «aniquilación de la violencia estatal», imperante dentro de un estado de derecho o agudizada en un estado excepción. La definición que hará Sorel, y que recupera Benjamin en su ensayo, es clarificadora en cuanto a los objetivos y lo que persigue la huelga general proletaria:

«Esta huelga general anuncia claramente su indiferencia con respecto al beneficio material de la conquista, al declarar que quiere suprimir el Estado; el Estado fue ciertamente... la razón de existencia de los grupos dominantes que se llevan el provecho de todas las empresas cuya carga soporta el conjunto...». Mientras que la primera forma de la suspensión del trabajo es violenta, puesto que provoca una modificación exterior de las condiciones de trabajo, la segunda es, como medio puro, no violenta. $Y$ es que ella no ocurre con la predisposición de reanudar el trabajo tras

\footnotetext{
${ }^{6}$ Hay que señalar que la noción de huelga general «es una idea muy antigua» —advertirá Carlos Pérez López citando a Jean Maitron. Incluso se considera que «el retiro de los plebeyos en el monte Aventino, en el año 493 a.C» podría ser una manifestación practica temprana del intento a huelga. Por otra parte, Sorel —afirma López-, retomará «la teoría de la huelga general producida por sus amigos sindicalistas revolucionarios a comienzos del siglo xx y la confronta con una versión que circula "por arriba", en las interpretaciones del marxismo que polarizaba al socialismo en dos corrientes incompatibles: la reformista y la revolucionaria" (Pérez López 2016, 45); en este escenario la huelga general proletaria sería de modo evidente la de tendencia revolucionaria.

${ }^{7}$ Benjamin en su artículo «Fuerza de Ley: el "fundamento místico de la autoridad"», hace una lectura del ensayo de Benjamin al que le critica por usar la noción de pureza, siendo que, para Derrida, nada es puro. Esta lectura por parte de Derrida omite que la pureza en Benjamin es un nombre técnico.
} 
concesiones externas y unas modificaciones cualesquiera de las condiciones laborales, sino con la resolución de reanudar solo un trabajo completamente modificado, no forzado por el Estado, subversión que este tipo de huelga, más que provocar, lleva a cabo (BENJAMIN, 2006, 15)

Algunas lecturas sobre este texto concluyen que la huelga general proletaria está en correlación con un tipo de anarquismo. Sin embargo, desde nuestra lectura, entendemos más bien que este tipo de huelga, tiene como objetivo articular una nueva forma de interacción, con un aire revolucionario en alguna sintonía con lo anárquico. Pues esta busca, dentro de la tradición de los oprimidos, contar otra historia y no simplemente instaurar un derecho, como sería el caso de la huelga política. Benjamin esquematizará las diferencias de estas dos huelgas a través de una huelga de médicos que aconteció en ciudades alemanas, en la que el filósofo identifica «de la manera más repugnante, el empleo inescrupuloso de la violencia», debido a que esta clase profesional no tiene «el más mínimo intento de resistencia». Lo que determina la huelga general proletaria es, junto con la idea revolucionaria, «la chance de que un día la humanidad mire al derecho y a su violencia como un objeto en desuso, pasado, obsoleto» (PÉREZ LÓPEZ, 2016, 83) y que se propicie o se abra «un tiempo revolucionario», que este pronto a venir.

Es pertinente, para concluir este apartado, preguntarnos entonces ¿cuándo o cómo acontece esta huelga general proletaria? $\mathrm{O}$ de qué manera accedemos al verdadero estado de excepción. Al respecto, y dentro de la imposibilidad de señalar un estadio concreto en el que se materialice la Huelga general proletaria, hemos de señalar que, a nuestro entender, esta existe y se dará en un momento del provenir. $\mathrm{O}$, tal vez, en el tiempo de una historia posible «que se corresponda» con las de los oprimidos superando lo que hasta ahora conocemos como «la norma histórica». De cualquier manera, creemos que esta huelga general proletaria, constantemente se actualiza a través de las revoluciones, que nos permiten pensar otra forma de historia y la inminencia de que esta acontezca. 


\section{La huelga general como mito y la irrupción del arte moderno en su configuración.}

Si de algo adolece el ensayo de hace Walter Benjamin, Para una crítica de la violencia, es la escasa discusión que le otorga a la teoría de los mitos, que realiza Sorel. Esta como un camino para comprender la huelga general proletaria basándose en la concepción de que es un hecho nunca acontecido. Esta se origina bajo la perspectiva de que una sustracción (sin violencia/ pura), permitirá destruir un sistema opresor por abandono. Lo que deja al descubierto la capacidad revolucionaria del trabajador. Esto, según lo advierte Rancière, es la primera imagen que sostiene el mito de la huelga general ${ }^{8}$. La noción de abandono, por otra parte, nos remite a una toma de conciencia por parte del sujeto que decide sustraerse del trabajo y paralizarlo por medio de la huelga general. Estos trabajadores — señala López- están «al borde la inconciencia del inocente ofrecido en sacrificio» (PÉREZ LÓPEZ, 2016, 52) y requieren de una «chispa» que les permita entrar en conciencia para construirse como sujeto activo en la emergente huelga general proletaria. Para llegar a esa chispa se requerirá «de un orador que prenda la llama».

Para Sorel el mito se reactualiza a partir de los cuestionamientos entre lo real y no real en la «promesa del socialismo». Aquí resurge el mito, pero esta vez «asociado al fantasma de la huelga general» (PÉREZ LÓPEZ, 2016, 59), sostenida por un «imaginario común» que deja al descubierto la trascendencia en los aspectos sociales y revolucionarios de esta. Se asume, por lo tanto, el potencial de la huelga de paralizar la historia (de la producción y el progreso), pues en el abandono o la cesación de trabajo se cancela la forma actual de concebir la historia. Se abre, por lo tanto, un espacio hacia algo "otro", hacia «trayectorias e imágenes que circulan en diferentes planos. En todos ellos, la huelga general parece ser un categoría de inminencia» (PÉREZ LÓPEZ, 2016, 74).

\footnotetext{
8 Las reflexiones entre Rancière y Gauny profundizan mucho más en la construcción del mito de la huelga general. Se remontan a «la deserción de los esclavos de Babilonia en la construcción del edificio monstruoso» que representaría la unión "demoniaca entre el dios Moloch y la torre de Babel», pues estos temen por la caída de esta.
} 
Ahora bien, volviendo a la idea de «toma de conciencia», es en ese espacio donde nosotros identificamos, siguiendo los trabajos de Benjamin, un choque que genera la activación de una consciencia política para encaminarse a la huelga general. Es decir, al asumir la huelga como mito, nos posicionamos en un estadio previo a esta, que es donde el trabajador accede, de modo particular, a otra experiencia, gracias a un shock. El trabajo que hace el investigador Pérez López, aunque no ve el shock en el sentido benjaminiano, señala de la siguiente manera la posibilidad de que emerja una conciencia de sustracción y paralización del trabajo, derivada de lo que él señala como una «chispa»:

\begin{abstract}
Bastaría solo con que fuesen conscientes para sustraerse al horrible fin de la libertad. La activación de esa conciencia puede depender de un orador que la ilumine, pero también puede darse en la exterioridad irregular del paseante que habla en voz alta y propaga esta imagen gracias a la eventual curiosidad de otro obrero dispuesto a prestarle atención. La chispa que enciende la conciencia viene a ser la imagen de esa inminencia, la posibilidad de que aquella se propague y circule (PÉREZ LÓPEZ, 2016, 74)
\end{abstract}

No es del todo improbable, que Benjamin considerase que un camino hacia la huelga general proletaria se encuentre determinado por un hecho previo a esta, residente en el individuo desde su particularidad. Es decir, para llegar a la comprensión o toma de conciencia respecto a la situación histórica, y de violencia, es necesario que el sujeto visualice la imagen (mítica si se quiere) de que la huelga es probable e inminente. Pero no basta con eso, el sujeto debe tomar conciencia, además, de que esta huelga pretende subvertir un orden teleológico de la historia para acceder a otra forma en que esta se articule. Uno de esos caminos, por lo tanto, estaría representado por medio de la experiencia moderna de la creación artística. En cuanto el arte moderno se concibe como una instancia creativa, experiencial y política.

Dicho lo anterior, deberíamos considerar, entonces, que el ensayo de Benjamin, Para una crítica de la violencia, es el resultado de reflexiones tempranas que el filósofo irá desarrollando en los trabajos posteriores. Estos escritos 
posteriores, aunque no siempre son enfocados desde una perspectiva política, como lo es Para una crítica de la violencia de 1921, sí establecen diálogos por medio de conceptos o ideas dentro del contexto en el que Benjamin los concibe. De esa manera, su producción política ${ }^{9}$ más importante es la que se publica de manera póstuma con el nombre de Tesis de la filosofía de la historia, trabajo que, a pesar de su complejidad, establece ideas definidas en torno a los temas que aborda. Señalamos esto a propósito de un hecho que nos permite pensar y establecer una relación entre elementos artísticos y políticos dentro del pensamiento benjaminiano.

Benjamin, después de leer a Carl Schmitt, y su libro Teología política del año 1922, queda al tanto de la noción que tiene este sobre la soberanía y el estado de excepción. Por consiguiente, prepara una respuesta en dos sentidos: le envía una carta y un libro de regalo. Sin embargo, la respuesta más importante a lo que plantea Schmitt, no será el contenido de la carta que, básicamente es una misiva formal y cordial. La respuesta al problema y tema político que plantea el jurista la hace Benjamin a través del libro que le envía de regalo:

\begin{abstract}
Muy estimado Señor Profesor: en estos días recibirá usted de la editorial un ejemplar de mi libro El origen del drama barroco alemán (...) Usted notará muy pronto cuánto debe a usted mi libro en la exposición del concepto de soberanía en el siglo XVII. Tal vez me permita decirle además que también sus obras posteriores, ante todo de la Dictadura he deducido una confirmación de mis modos de investigación filosófico-artísticos.
\end{abstract}

Es decir, la respuesta a la temática política que se plantea en los trabajos de Schmitt, tiene una respuesta desde la obra de arte, entendiendo que este es el tema en el que incursiona Benjamin en este libro del año 1925 (pero concebido algunos años antes). Hemos señalado este hecho para reafirmar y dibujar el ingreso de la obra de arte dentro del entramado que hasta hemos realizado a través de los conceptos e ideas de huelga general proletaria y (verdadero) estado de excepción.

\footnotetext{
${ }^{9}$ Los textos políticos esenciales de Benjamin son tres: Para una crítica de la violencia, El fragmento teológico-político y Las tesis sobre historia. En, Para una crítica de la violencia, es donde esboza de manera más evidente y bosquejada la idea de huelga general y el proyecto filosófico-político global dentro de su producción y reflexión.
} 
Ahora bien, esto mismo es señalado por Bolívar Echeverría, quien afirma que Benjamin «es un convencido de que en la dimensión discursiva, lo político se juega -y de manera a veces incluso más decisiva - en escenarios aparentemente ajenos al de la política propiamente dicha» (ECHEVERRÍA, 2003, 9). Queremos introducir, con lo último que se ha señalado, la noción de que el arte (siguiendo lo que desarrolla Benjamin en su libro de 1936) constituye muchas veces un shock que permite, a nuestro entender, desbaratar la estrategia capitalista o bien provocar la toma de conciencia en sujetos que no advierten la forma violenta en que el estado de derecho se ha constituido por siglos, pero que tal situación se puede desbaratar por medio de la paralización universal del trabajo que sería la huelga general proletaria, cuyo acontecer es inminente.

En definitiva, y para sintetizar lo que se ha expuesto, el camino que hemos trazado hasta aquí no responde más que una lectura particular propiciada por una hipótesis en la que hacemos dialogar distintos conceptos de la obra de Walter Benjamin. Esto nos ha dado por resultado, a diferencia de lo que en principio planteamos, un viaje al origen esencial de la huelga. Es decir, si en principio nuestro interés se asentaba en relacionar huelga y estado de excepción poniendo de relieve que una contenía a la otra y viceversa. Tal planteamiento, lo propusimos para poder acercarnos o esbozar alguna idea sobre la manera en que podríamos ingresar a un nuevo orden histórico de las cosas; aquella otra forma histórica (o verdadero estado de excepción) que Benjamin propone en la tesis VIII. Suponiendo, claro, que el camino más evidente era la huelga general. Sin embargo, al profundizar en la huelga general proletaria en la que se detiene Sorel y Benjamin, advertimos que las características de esta y su conformación no han llegado a ser un hecho concreto. Tal situación nos llevó a indagar en la configuración del sujeto antes de conformar la huelga. Es aquí donde nos limitamos a señalar la repercusión que tiene el arte, como manifestación política, que coadyuva a que el sujeto, desde su particularidad, comprenda la posibilidad de construir una historia distinta al estado de excepción permanente, en el que viven los oprimidos dentro de esta sociedad. Coincidimos, por lo tanto, con la afirmación de que la huelga general proletaria «era menos de lo que anunciaba, esto es, la suspensión universal del trabajo que nunca aconteció», 
pero al mismo tiempo era mucho más de lo que «se mostraba de ella, es decir, la preparación real de su advenimiento inminente» (PÉREZ LÓPEZ, 2016, 76).

\section{REFERÊNCIAS}

AGAMBEN, Giorgio. 2010. Estado de excepción: homo sacer II, 1. Traducido por Antonio Gimeno Cuspinera. Valencia: Pre-textos.

BENJAMIN, Walter. 2003. La obra de arte en la época de su reproductibilidad técnica [urtext]. Traducido por Andrés E Weikert. México: Editorial Itaca.

Oyarzun. Versión electrónica. 2006. Para una crítica de la violencia. Traducido por Pablo . 2009. La dialéctica en suspenso. Fragmentos sobre la historia. Traducido por Pablo Oyarzun. Santiago: LOM

CUMSILLE, Kamal. 2007. «Shock y Estado de excepción: Arte y política moderna, Baudelaire y Benjamin». A Parte Rei Revista de Filosofía 51: 1-7.

LÖWY, Michael. 2012. Walter Benjamin: aviso de incendio. Una lectura de las tesis "Sobre el concepto de historia". Buenos Aires: Fondo de Cultura Económica.

LUCCA, Juan. 2009. «Walter Benjamin y Carl Schmitt. Palabras cruzadas de un diálogo mudo en un tiempo agitado». Revista de Filosofía 62: 87-111.

MATE, Reyes. 2006. Medianoche en la historia: comentarios a las tesis de Walter Benjamin «Sobre el concepto de historia». Colección Estructuras y procesos. Madrid: Editorial Trotta.

PÉREZ LÓPEZ, Carlos. 2016. La huelga general como problema filosófico: Walter Benjamin y Georges Sorel. Santiago de Chile: Ediciones, Metales Pesados

RANCIÈRE, Jacques. 2010. La noche de los proletarios: archivo del sueño obrero. Buenos Aires: Tinta Limon.

SOREL, Georges. 1973. Reflexiones sobre la violencia. Traducción Luis Alberto Ruiz. Buenos Aires: La pléyade. 